\title{
Studying Brown Adipose Tissue in a Human in vitro Context
}

\author{
Isabella Samuelson ${ }^{1,2 *}$ and Antonio Vidal-Puig ${ }^{1,2}$ \\ ${ }^{1}$ Metabolic Research Laboratories, University of Cambridge, Cambridge, United Kingdom, ${ }^{2}$ Department of Cellular Genetics, \\ Wellcome Sanger Institute (WT), Hinxton, United Kingdom
}

OPEN ACCESS

Edited by:

Jiri Jiracek,

Academy of Sciences of the Czech

Republic (ASCR), Czechia

Reviewed by:

P. Trayhurn,

University of Liverpool,

United Kingdom

Alexander W. Fischer,

Harvard University, United States

*Correspondence:

Isabella Samuelson

iks25@cam.ac.uk

Specialty section:

This article was submitted to

Experimental Endocrinology,

a section of the journal

Frontiers in Endocrinology

Received: 29 June 2020

Accepted: 04 August 2020

Published: 15 September 2020

Citation:

Samuelson I and Vidal-Puig A (2020) Studying Brown Adipose Tissue in a

Human in vitro Context.

Front. Endocrinol. 11:629.

doi: 10.3389/fendo.2020.00629
New treatments for obesity and associated metabolic disease are increasingly warranted with the growth of the obesity pandemic. Brown adipose tissue (BAT) may represent a promising therapeutic target to treat obesity, as this tissue has been shown to regulate energy expenditure through non-shivering thermogenesis. Three different strategies could be employed for therapeutic targeting of human thermogenic adipocytes: increasing BAT mass through stimulation of BAT progenitors, increasing BAT function through regulatory pathways, and increasing WAT browning through promotion of beige adipocyte formation. However, these strategies require deeper understanding of human brown and beige adipocytes. While murine studies have greatly increased our understanding of BAT, it is becoming clear that human BAT does not exactly resemble that of the mouse, highlighting the need for human in vitro models of brown adipocytes. Several different human brown adipocyte models will be discussed here, along with the potential to improve brown adipocyte culture through recreation of the BAT microenvironment.

Keywords: brown adipose tissue (BAT), 3D culture, HPSC model, obesity, thermogenic adipocyte differentiation, brown adipogenesis, human adipocytes, beige adipocyte

\section{THE OBESITY PANDEMIC}

Obesity, defined as excessive fat accumulation, has reached pandemic proportions. Among adults, more than 650 million were obese in 2016 (BMI $\geq 30$ ), with over 340 million obese or overweight $(\mathrm{BMI} \geq 25)$ children and adolescents (1). Obesity is associated with the metabolic syndrome, which comprises a cluster of metabolic abnormalities including insulin resistance, obesity, dyslipidemia, and hypertension, which are risk factors for cardiovascular disease and diabetes (2). Furthermore, obesity is a risk factor for cancer and musculoskeletal disorders, including osteoarthritis, among other diseases $(3,4)$. Thus, obesity places a substantial burden on society and healthcare systems and despite extensive research into the mechanisms controlling energy balance, body weight, and appetite, safe and effective measures to treat and prevent obesity are lacking.

\section{BROWN ADIPOSE TISSUE}

Brown adipose tissue (BAT) has become a focus area for weight loss therapies. This tissue, evolved to help maintain core body temperature, is able to burn nutrients as heat, thereby increasing total energy expenditure (5). This process is known as non-shivering thermogenesis and is mediated by the mitochondrial uncoupling protein1 (UCP1), and BAT activation leads to increased energy expenditure and decreased metabolic efficiency. 
BAT has been extensively studied in the mouse, and these studies have revealed an important role for BAT in the control of energy balance and whole-body metabolism. BAT activation has, for instance, been shown to positively influence plasma triglyceride and cholesterol levels, atherosclerosis development, glucose tolerance, insulin sensitivity, and hepatic steatosis, in addition to promoting weight loss (6-9).

\section{BEIGE ADIPOSE TISSUE}

Another type of adipose tissue (AT), which has received considerable attention for the treatment of obesity, is beige AT, which arises from white adipose tissue (WAT) browning. WAT browning is characterized by the presence of multilocular, brown adipocyte (BA)-marker expressing adipocytes within WAT-socalled beige or brite adipocytes. Beige adipocytes can appear in WAT, mainly subcutaneous (sc)WAT, after cold exposure, hypercaloric diet, $\beta$-adrenergic stimulation, and exercise (10), and are believed to influence metabolism similarly to BAT (11, 12). Although beige adipocytes show high expression of UCP1 (13), they are believed to originate from different lineages than BAs (14), although this is still debated.

\section{BAT IN HUMANS - BEIGE OR BROWN?}

In humans, BAT mass and prevalence have been found to correlate negatively with BMI, diabetes status, and glucose plasma levels (15), and cold-induced BAT activation also had beneficial metabolic effects and induced weight loss $(16,17)$, suggesting that human and murine BAT may have similar metabolic roles. It has been estimated that adult lean men may have around $330 \mathrm{ml}$ of cold-inducible BAT, with lower volumes (around $130 \mathrm{ml}$ ) found in obese men (18). Furthermore, seasonal changes in WAT thermogenic gene expression have been described (19), suggesting that human WAT has the capacity for browning, although the impact of browning on whole-body metabolism is unclear.

Based on this ability of BAT to increase energy expenditure and improve metabolic function, BAT seems a likely target for the development of obesity therapeutics. Importantly, the efficacy of these therapeutics depends on whether activation of BAT in adults is able to significantly influence energy expenditure. Cold exposure in adults is able to increase energy expenditure varying from $20 \mathrm{kcal} /$ day (20) to more than $100 \mathrm{kcal} /$ day (17), and this can be increased further through chronic cold adaptation or increased BAT mass $(17,18)$.

A growing body of research is focusing on identifying genes and molecules able to influence BAT biology; however, one question is how well the findings in mice translate to humans. First, there is debate around whether human BAT resembles murine brown or beige AT more closely. Analyses of human neck fat have revealed that on a molecular level, deep neck fat mostly resembles that of murine canonical BAT, whereas the superficial neck fat may be more white or beige (21). In a study characterizing beige adipocytes, $\mathrm{Wu}$ et al. (13) generated murine brown and beige adipocyte gene expression signatures.
Comparing these signatures with human supraclavicular BAT, they found that human BAT is closer to murine beige than brown AT. A similar study comparing murine AT gene signatures with human BAT from multiple anatomical locations came to the same conclusion (22). In contrast, a recent study suggests that when mice are physiologically humanized, meaning housed at thermoneutrality, fed a "Western diet," and examined at 911 months old, their BAT becomes comparable with human supraclavicular BAT (23), although this conclusion has raised some discussion (24). This debate may likely be solved as the composition of human and murine brown and beige AT is better characterized with the help of more sophisticated techniques such as single-cell RNA sequencing. Owing to discrepancies between mRNA and protein expression levels of certain genes, including UCP1 (25), purely transcriptomic analysis of different BA populations may not be adequate to fully characterize their identity and thermogenic potential. To this end, protein analysis and functional characterization, including $\beta$-adrenergic response and bioenergetics, are required.

Second, whereas treatment with $\beta_{3}$-adrenergic receptor $\left(\beta_{3} \mathrm{AR}\right)$ agonists in mice is able to selectively stimulate BAT and protect against diet-induced obesity, these effects are not seen in humans at doses low enough to prevent cardiovascular side effects $(26,27)$. Interestingly, a recent study found that the $\beta_{1} \mathrm{AR}$ may be the predominant $\beta \mathrm{AR}$ in human BAT (28), highlighting another confounding issue when translating murine BAT studies to human biology. Other attempts to selectively activate thermogenesis through sympathomimetic drugs or mitochondrial uncouplers have not been successful (26). Thus, to achieve selective and safe activation of BAT in humans, we may need to look beyond adrenergic stimulation or uncoupling mechanisms and instead focus on alternative pathways to target BAT biology. Specifically, rather than focusing exclusively on increasing the activation of existing BAT, other strategies could include increasing BAT mass through differentiation of progenitors, or increasing thermogenic activity in WAT through WAT browning.

\section{STRATEGIES FOR THE TARGETING OF HUMAN BAT}

Increased energy expenditure in humans through BAT thermogenesis could be achieved by (a) increasing functional BAT mass, (b) increasing BAT activity, and (c) increasing WAT browning.

\section{Increased BAT Mass/Recruitment}

Increased BAT mass could be achieved through the recruitment of BA progenitors. As mentioned earlier, decreased BAT mass is found in obese individuals-the very individuals the BAT therapeutics are intended for-and thus even if a method to selectively activate BAT was found, this may be of limited use to obese recipients. However, BAT activity can be enhanced through cold acclimation even in obese individuals (29), suggesting the presence of BA precursors, the differentiation of which may be promoted given appropriate stimulation. The development of 
targeted approaches to induce differentiation of BA progenitors requires in-depth knowledge of the developmental mechanisms of human BAT, which currently does not exist. Furthermore, newly differentiated BAs would still require activation through sympathetic or alternative pathways.

\section{Increased BAT Activity}

Increased BAT activity, as discussed earlier, has been challenging to achieve without cardiovascular side effects. However, several molecules have been identified as possible therapeutic targets for the activation of BAT, reviewed in (30). First, members of the bone morphogenetic protein (BMP) family have been reported to play roles in BAT or WAT browning, mainly using murine models. These include, among others, BMP4, which may promote browning of WAT $(31,32)$, BMP7, which can promote BAT development and activation (33) as well as white adipocyte (WA) browning (31), and BMP8B, which was found to increase the adrenergic response of BAT and induce scWAT browning $(34,35)$. In addition, factors released from heart, muscle, liver, and immune cells have been shown to regulate thermogenesis (30). Although these studies reveal several candidate targets, most of them have been conducted in murine models or in vitro, and tangible therapeutics are still far from a reality.

\section{Increased WAT Browning}

Finally, increased WAT browning is a strategy which may have substantial potential given the high abundance of WAT compared with BAT. However, as for BAs, the safe activation of beige adipocytes likely requires identification of specific molecular pathways to induce WAT browning that do not rely directly on adrenergic or uncoupling actions. These include fibroblast growth factor 21 (FGF21), which may regulate browning of scWAT through activation of peroxisome proliferator-activated receptor gamma coactivator 1-alpha (PGC1 $\alpha)$ (36), as well as through UCP1-independent mechanisms (37). Another potential molecule is irisin, which may activate UCP1 in scWAT via p38-MAPK/ERK pathways (38), although the levels of circulating irisin and its metabolic effects in humans are debated $(39,40)$. In addition, more studies on human WAT browning are necessary to determine whether this process is able to influence whole-body energy expenditure in a notable manner.

The three strategies presented earlier are all limited by the lack of knowledge regarding human thermogenic adipocyte development and function. This lack of knowledge stems principally from the lack of robust in vitro models of human BAs.

\section{IN VITRO MODELS OF HUMAN BAT}

Models of human BAs have been developed by several different groups and from starting material such as primary BAT, fibroblasts, muscle cells, adult stem cells, and pluripotent stem cells, among others. Some of these models are discussed later and represented in Figure 1.

\section{Primary Cell-Derived BA Models}

The study of human primary BAT is limited by the scarceness of the disperse tissue and the need for invasive procedures to obtain biopsies. Nonetheless, several in vitro BA models derived from primary human BAT do exist. If one does have access to primary BAT, preadipocytes from non-viable human fetal interscapular BAT can be cultured and differentiated in vitro (41); however, these are not stable cell lines and are thus not a viable long-term solution for the study of human BAT. To this end, immortalized human BA lines may be of more use. One of these is the PAZ6 cell line from immortalized infant BAT vascular stromal cells (42). These cells readily differentiate to BAs and respond to $\beta$-adrenergic stimulation; however, the differentiation potential of these cells declines with increasing passage number, significantly limiting their experimental value. Similarly, subcloned immortalized BA-like cell lines have been generated from adult supraclavicular AT stromal vascular fraction (SVF) (43) and neck AT SVF (44). In addition, a more heterogeneous BA-like cell model was created from adult deep neck AT SVF without subcloning (45). These BA cell models demonstrated expression of $U C P 1$, lipolytic response to cAMP (43), and increased oxygen consumption in response to $\beta$-adrenergic stimulation $(44,45)$, and two of the lines were maintained for at least 20 passages $(44,45)$. These cell lines thus represent useful models for the study of human BAT. However, their generation does require access to primary tissue, and they, therefore, come from limited starting material, which means that these cell lines will deplete eventually.

Rather than using primary BAT, BA-like cells can be directly generated from human dermal fibroblasts, eliminating the need for invasive biopsies. One study demonstrated that overexpression of CCAAT/enhancer binding protein beta $(C E B P B)$ and avian myelocytomatosis viral oncogene homolog $(C-M Y C)$, coupled with an adipogenic cocktail, induced conversion of human fibroblasts to BA-like cells (46). Another study demonstrated the direct conversion of primary fibroblasts into BA-like cells with only chemical compounds, without using gene transfer (47). These compounds included rosiglitazone, which is an agonist of peroxisome proliferator-activated receptor gamma (PPAR $\gamma$ ), and known to promote browning (48).

Alternatively, BA-like cells were shown to be differentiated from fetal and adult muscle progenitor cells expressing CD34, a marker of hematopoietic progenitors as well as vascular endothelial progenitors (49), thus supporting the common lineage of skeletal muscle and BAT. This technique does require invasive biopsies; however, muscle tissue may be more accessible than BAT.

In addition to primary tissue access, a limitation of the aforementioned models is the inherent risk of cell depletion. Thus, to circumvent this limitation, cell lines can be generated from human stem cells.

\section{Generation of Adipocytes From Stem Cells Multipotent Stem Cell-Derived BAs}

Multipotent mesenchymal stem cells (MSCs) are adult stem cells able to differentiate into a range of mesodermal cell types including adipocytes, and can be isolated from several tissues 


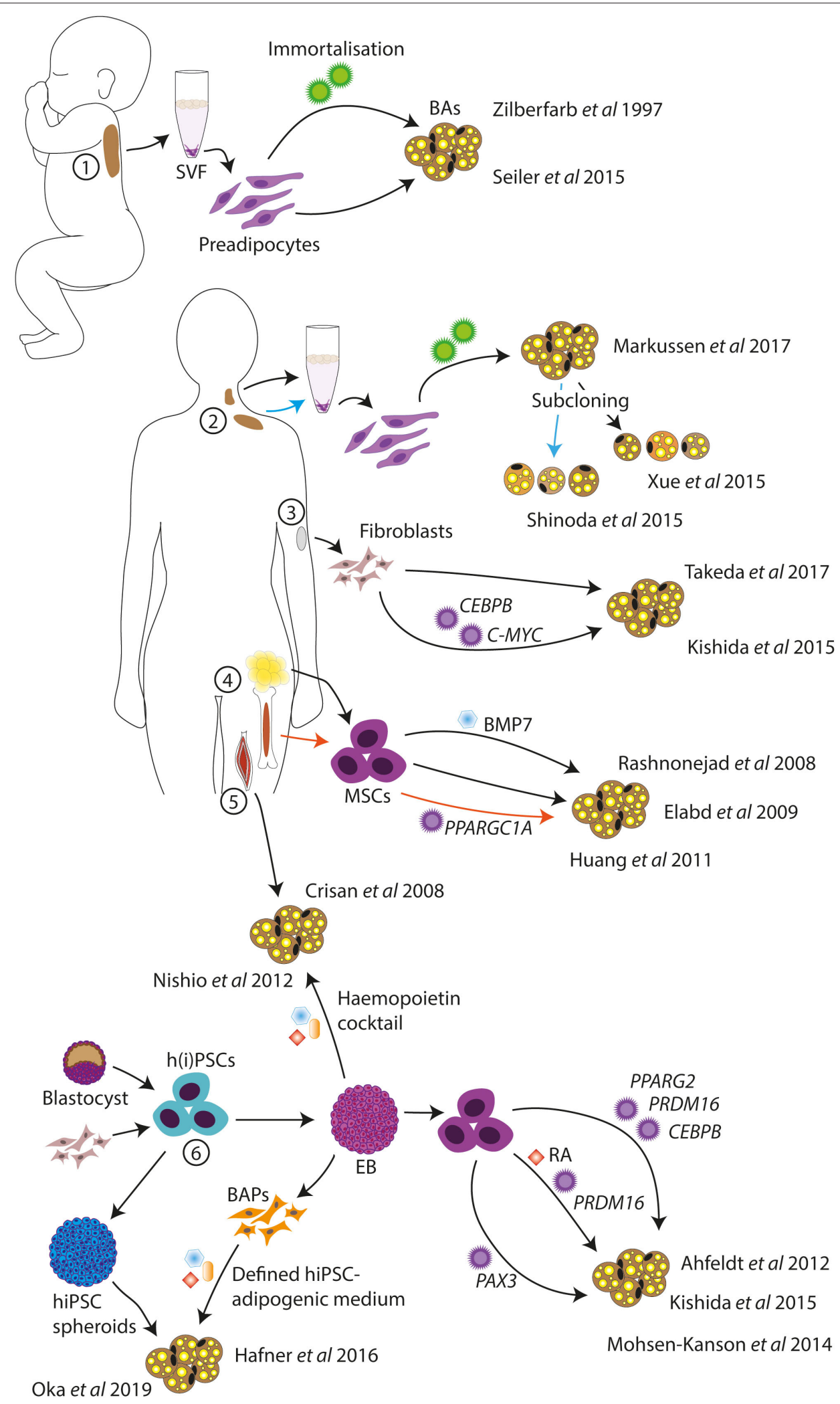

FIGURE 1 | Schematic of in vitro human BA models. Human BA models have been generated from infant BAT (1), deep neck and supraclavicular BAT (2), dermal fibroblasts (3), MSCs from AT and bone marrow (4), skeletal muscle progenitor cells (5), and hPSCs (6). Standard adipogenic cocktails or variations were used for the differentiations unless otherwise indicated. SVF, stromal vascular fraction; BAs, brown adipocytes; MSCs, mesenchymal stem cells; h(i)PSCs, human (induced) pluripotent stem cells; BAPs, BA progenitors; EB, embryoid body; RA, retinoic acid. 
such as bone marrow, blood, and AT (50). MSCs isolated from human AT (termed human multipotent adipose-derived stem (hMADS) cells) could be differentiated to BA-like cells when stimulated with a PPAR $\gamma$ agonist on top of an adipogenic cocktail (51), and with the addition of BMP7 (52). Similarly, bone marrow-derived MSCs may also be able to differentiate to BAlike cells when overexpressing PPARGC1A (53). Thus, MSCs are a useful tool for the generation of BA-like cells, and the selfrenewal ability of MSCs gives unlimited starting material, at least theoretically. As an alternative to MSCs, human pluripotent stem cells (hPSCs) have been used for the generation of BA cell models.

\section{Pluripotent Stem Cell-Derived BAs}

hPSCs are advantageous for the generation of cell models for several reasons, including that they are relatively easy to genetically engineer. Because of their pluripotency and the fact that protocols exist to differentiate hPSCs into a wide range of cell types (54), it is possible to generate isogenic disease models of cells from all three embryonic germ layers. Furthermore, the use of human induced (hi)PSCs, which are reprogrammed from human somatic cells (55), enables the generation of disease models with patient-specific genetic backgrounds.

Some studies have demonstrated the generation of adipocytes from hPSCs. Mature white and brown adipocytes were generated from hPSCs through transfection with lentiviral constructs to overexpress PPARG2 or PPARG2, CEBPB, and PRDM16, respectively (56). In this study, hPSCs were first differentiated into MSCs using embryoid bodies (EBs), after which transgene expression was induced and an adipogenic cocktail was given. A similar method was used to generate BAs from hiPSCs through transduction of PRDM16 (46). Here, EBs were generated in the presence of retinoic acid (RA), to obtain more myoblast-like cells (57), accounting for the reduced transgene requirement. Overexpression of transgenes in hPSCs is an efficient method for the generation and study of mature cell types (58-60), although it can be argued that this method of differentiation may not follow natural developmental steps and may instead shunt some of these pathways. Thus, this technique may not be optimal for the generation of a human BA model for the study of BA development.

Another group demonstrated the differentiation of BA progenitors from hiPSCs through EB formation and adipogenic cocktails (61). The differentiation efficiency was reported to be low but could be improved through overexpression of paired box 3 (PAX3), a marker of BA progenitors (61). A similar method was used to generate $\mathrm{BA}$ progenitors from hiPSCs without gene transfer, although this study reports higher differentiation yield, possibly due to inhibition of TGF $\beta$ signaling (62). TGF $\beta$ signaling has been shown to induce the conversion of endothelial cells to a more mesenchymal phenotype (63), and these findings, therefore, suggest an important role of endothelial cells in the adipogenic niche. The generation of BA-like cells from hPSCs using EB formation and a hemopoietin cocktail has also been reported (64), which may suggest that the developmental origin of BAs is more diverse than previously believed. Finally, BA-like cells were generated from hiPSCs in cytokine-free medium through the formation of spheroids (65), although the molecular mechanisms of this differentiation method remain unclear.

Although hPSCs can, in theory, give rise to any cell type of the human body, their immature/embryonic state means that it can be difficult to generate mature cell types from hPSCs without forward programming. This issue does not arise when using BA models derived from primary adult AT. As an alternative, hPSC-derived BAs can be matured in vivo by transplantation into mice, which is also a method of functional validation of the generated BAs $(46,64,65)$. Currently, transplantation in vivo may be the best technique for recapitulation of the physiological BAT microenvironment; however, advances are also being made toward recreating BAT in vitro using $3 \mathrm{D}$ cell cultures.

\section{RECAPITULATING THE BA MICROENVIRONMENT IN VITRO}

Long-term culture of adipocytes in vitro can be difficult because of the buoyancy of mature, lipid-laden adipocytes, which makes their attachment to $2 \mathrm{D}$ cell culture plates difficult (66). Furthermore, true understanding of BAT biology requires recapitulation of the in vivo BA microenvironment-the BA niche. In vivo, BAT is a highly vascularized and innervated tissue, containing not only BAs but also endothelial cells, fibroblasts, progenitor cells, and immune cells $(5,30)$. Furthermore, the extracellular matrix (ECM) of the AT is vital as a structural scaffold and to facilitate adipocyte differentiation, AT expansion, mechanotransduction, and biomolecule signaling (67-69).

\section{Culturing BAs in 3D}

A first step toward recapitulating BAT in vitro may be to use 3D cultures, which can provide mechanical support, allow physiological cell organization, and prevent loss of mature adipocytes (70). 3D culture techniques are successful for WA culture (71), allowing in vitro culture of unilocular primary mature human WAs $(72,73)$. Unilocular WAs can also develop in vitro when differentiated in spheroids (74-76).

Fewer 3D culture systems have been tested on BAs, potentially because the focus is still on the generation of useful BA cell models rather than their further development. Using hanging drop spheroid formation followed by culture in ultra-low attachment plates, Klingelhutz et al. (76) demonstrated improved differentiation of murine BAT SVF compared with 2D. Alginate microstrands have also been used for the differentiation of BAs from murine embryonic stem cells and brown preadipocytes (77). Using 3D printed hyaluronic acid/gelatin gels, Kuss et al. (78) showed that whereas immortalized human WA progenitors prefer soft gels, immortalized human BA progenitors prefer stiffer gels, suggesting that developments within WA 3D culture methods cannot necessarily be directly translated to BA cultures.

\section{ECM and Vascularization}

The next step toward recapitulating BAT in vitro is the addition of ECM and supporting cell types. It is possible to generate 3D scaffolds from ECM proteins such as collagens and glycoproteins (71), or to incorporate ECM components into synthetic hydrogels $(72,79)$. However, to recreate the human BAT ECM 
in vitro, its composition must first be fully characterized in vivo, in both physiological and pathophysiological states.

For the generation of functional models of AT, vascularization is an important parameter, which can also aid engraftment in vivo during transplantation studies, as shown (74). Angiogenic signals, including VEGF (vascular endothelial growth factor), play important roles not only in BAT function but also in the development of BAs (80-83). For instance, VEGFA is able to trigger local angiogenesis in BAT, leading to brown adipogenesis and upregulation of Ucp1 and $\operatorname{Pgc1\alpha }(81)$. Vascularized (white) adipocyte spheroids can be generated using AT SVF, which contains endothelial cells as well as preadipocytes, or by coculturing adipocytes with endothelial cells $(74,75)$. For the generation of vascularized human BA models, it may be advantageous to focus on coculture methods, to avoid the need for primary tissue. Similar challenges exist regarding the integration of immune cells into the BA cultures.

\section{DISCUSSION}

Our understanding of BAT in mice and humans has increased significantly over the last decade, and with this understanding, we are moving closer to the development of therapeutics targeting BAT for obesity treatment. One of the main limitations in the field is the limited understanding of human BAT, including its development and molecular signature. These questions are steadily being tackled through in-depth characterization of primary human BAT and the development of human in vitro BA models. Human BA models can be generated from immortalization of primary human BAs, from multipotent stem cells or hPSCs, among others, and each of these methods has

\section{REFERENCES}

1. World Health Organization. Obesity and Overweight. (2020). Available online at: https://www.who.int/news-room/fact-sheets/detail/obesity-andoverweight (accessed March 18, 2020).

2. Eckel RH, Grundy SM, Zimmet PZ. The metabolic syndrome. Lancet. (2005) 365:1415-28. doi: 10.1016/S0140-6736(05)66378-7

3. Kolb R, Sutterwala FS, Zhang W. Obesity and cancer: inflammation bridges the two. Curr Opin Pharmacol. (2016) 29:77-89. doi: 10.1016/j.coph.2016.07.005

4. King LK, March L, Anandacoomarasamy A. Obesity and osteoarthritis. Indian J Med Res. (2013) 138:185-93.

5. Cannon B, Nedergaard J. Brown adipose tissue: function and physiological significance. Physiol Rev. (2004) 84:277-359. doi: 10.1152/physrev.00015.2003

6. Bartelt A, Bruns OT, Reimer R, Hohenberg H, Ittrich H, Peldschus K, et al. Brown adipose tissue activity controls triglyceride clearance. Nat Med. (2011) 17:200-5. doi: 10.1038/nm.2297

7. Berbée JFP, Boon MR, Khedoe PPSJ, Bartelt A, Schlein C, Worthmann A, et al. Brown fat activation reduces hypercholesterolaemia and protects from atherosclerosis development. Nat Commun. (2015) 6:6356. doi: 10.1038/ncomms7356

8. Liu X, Zheng Z, Zhu X, Meng M, Li L, Shen Y, et al. Brown adipose tissue transplantation improves whole-body energy metabolism. Cell Res. (2013) 23:851-4. doi: 10.1038/cr.2013.64

9. Stanford KI, Middelbeek RJW, Townsend KL, An D, Nygaard EB, Hitchcox KM, et al. Brown adipose tissue regulates glucose homeostasis distinct advantages and limitations. For instance, in vitro models generated from adult BAT may represent the most mature BA models, whereas hPSC-derived BAs may be less mature unless forward programming is employed. In contrast, hPSCs are useful for disease modeling involving genetic engineering or patientspecific genetic backgrounds. Without a defined molecular signature for human BAT, lacking due to the heterogeneity and disperse anatomical distribution of BAT depots, it may, however, be challenging to determine which in vitro model is the best. Furthermore, as BAT does not exclusively consist of BAs, true recapitulation of this tissue involves modeling of the BAT microenvironment, including physiological BA organization in $3 \mathrm{D}$, crosstalk with supporting cells such as immune cells, vascularization, and an ECM to facilitate BA differentiation and cell-cell signaling.

The road toward the development of obesity therapeutics targeting human BAT thus has plenty of challenges. However, with continued development of BA models and characterization of the in vivo depots, these challenges may soon be overcome.

\section{AUTHOR CONTRIBUTIONS}

IS and AV-P wrote the manuscript. All authors contributed to the article and approved the submitted version.

\section{FUNDING}

The authors gratefully acknowledge support from the Medical Research Council (PO 4050281695), the British Heart Foundation (RG/12/13/29853), the European Research Council (669879), and Wellcome (109153/Z/15/Z). and insulin sensitivity. J Clin Invest. (2013) 123:215-23. doi: 10.1172/JCI6 2308

10. Dewal RS, Stanford KI. Effects of exercise on brown and beige adipocytes. Biochim Biophys Acta Mol Cell Biol Lipids. (2019) 1864:71-8. doi: 10.1016/j.bbalip.2018.04.013

11. Cohen P, Levy JD, Zhang Y, Frontini A, Kolodin DP, Svensson KJ, et al. Ablation of PRDM16 and beige adipose causes metabolic dysfunction and a subcutaneous to visceral fat switch. Cell. (2014) 156:304-16. doi: 10.1016/j.cell.2013.12.021

12. Seale P, Conroe HM, Estall J, Kajimura S, Frontini A, Ishibashi J, et al. Prdm16 determines the thermogenic program of subcutaneous white adipose tissue in mice. J Clin Invest. (2011) 121:96-105. doi: 10.1172/JCI44271

13. Wu J, Boström P, Sparks LM, Ye L, Choi JH, Giang A-H, et al. Beige adipocytes are a distinct type of thermogenic fat cell in mouse and human. Cell. (2012) 150:366-76. doi: 10.1016/j.cell.2012.05.016

14. Seale P, Bjork B, Yang W, Kajimura S, Chin S, Kuang S, et al. PRDM16 controls a brown fat/skeletal muscle switch. Nature. (2008) 454:9617. doi: 10.1038/nature07182

15. Cypess AM, Lehman S, Williams G, Tal I, Rodman D, Goldfine AB, et al. Identification and importance of brown adipose tissue in adult humans. $N$ Engl J Med. (2009) 360:1509-17. doi: 10.1056/NEJMoa0810780

16. Chondronikola M, Volpi E, Børsheim E, Porter C, Annamalai $\mathrm{P}$, Enerbäck $\mathrm{S}$, et al. Brown adipose tissue improves whole-body glucose homeostasis and insulin sensitivity in humans. Diabetes. (2014) 63:4089-99. doi: 10.2337/db14-0 746 
17. Yoneshiro T, Aita S, Matsushita M, Kayahara T, Kameya T, Kawai Y, et al. Recruited brown adipose tissue as an antiobesity agent in humans. J Clin Invest. (2013) 123:3404-8. doi: 10.1172/JCI67803

18. Leitner BP, Huang S, Brychta RJ, Duckworth CJ, Baskin AS, McGehee S, et al. Mapping of human brown adipose tissue in lean and obese young men. Proc Natl Acad Sci USA. (2017) 114:8649-54. doi: 10.1073/pnas.1705287114

19. Kern PA, Finlin BS, Zhu B, Rasouli N, McGehee RE, Westgate PM, et al. The effects of temperature and seasons on subcutaneous white adipose tissue in humans: evidence for thermogenic gene induction. J Clin Endocrinol Metab. (2014) 99:E2772-9. doi: 10.1210/jc.2014-2440

20. Kulterer OC, Niederstaetter L, Herz CT, Haug AR, Bileck A, Pils D, et al. The presence of active brown adipose tissue determines cold-induced energy expenditure and oxylipin profiles in humans. J Clin Endocrinol Metab. (2020) 105:2203-16. doi: 10.1210/clinem/dgaa183

21. Cypess AM, White AP, Vernochet C, Schulz TJ, Xue R, Sass CA, et al. Anatomical localization, gene expression profiling and functional characterization of adult human neck brown fat. Nat Med. (2013) 19:6359. doi: $10.1038 / \mathrm{nm} .3112$

22. Sharp LZ, Shinoda K, Ohno H, Scheel DW, Tomoda E, Ruiz L, et al. Human BAT possesses molecular signatures that resemble beige/brite cells. PLOS ONE. (2012) 7:e49452. doi: 10.1371/journal.pone.0049452

23. de Jong JMA, Sun W, Pires ND, Frontini A, Balaz M, Jespersen NZ, et al. Human brown adipose tissue is phenocopied by classical brown adipose tissue in physiologically humanized mice. Nat Metab. (2019) 1:83043. doi: 10.1038/s42255-019-0101-4

24. Kajimura S, Spiegelman BM. Confounding issues in the 'humanized' BAT of mice. Nat Metab. (2020) 2:303-4. doi: 10.1038/s42255-020-0192-y

25. Nedergaard J, Cannon B. UCP1 mRNA does not produce heat. Biochim Biophys Acta. (2013) 1831:943-9. doi: 10.1016/j.bbalip.2013.01.009

26. Whittle AJ, López M, Vidal-Puig A, Jia H, Lubetkin EI, Allison DB, et al. Using brown adipose tissue to treat obesity - the central issue. Trends $\mathrm{Mol} \mathrm{Med}$. (2011) 17:405-11. doi: 10.1016/j.molmed.2011.04.001

27. Cypess AM, Weiner LS, Roberts-Toler C, Franquet Elía E, Kessler SH, Kahn PA, et al. Activation of human brown adipose tissue by a $\beta 3$-adrenergic receptor agonist. Cell Metab. (2015) 21:33-8. doi: 10.1016/j.cmet.2014.12.009

28. Riis-Vestergaard MJ, Richelsen B, Bruun JM, Li W, Hansen JB, Pedersen SB. Beta-1 and not beta-3 adrenergic receptors may be the primary regulator of human brown adipocyte metabolism. J Clin Endocrinol Metab. (2020) 105:e994-1005. doi: 10.1210/clinem/dgz298

29. Hanssen MJW, van der Lans AAJJ, Brans B, Hoeks J, Jardon KMC, Schaart $\mathrm{G}$, et al. Short-term cold acclimation recruits brown adipose tissue in obese humans. Diabetes. (2016) 65:1179-89. doi: 10.2337/db15-1372

30. Carobbio S, Guénantin A-C, Samuelson I, Bahri M, Vidal-Puig A. Brown and beige fat: from molecules to physiology and pathophysiology. Biochim Biophys Acta Mol Cell Biol Lipids. (2019) 1864:37-50. doi: 10.1016/j.bbalip.2018.05.013

31. Elsen M, Raschke S, Tennagels N, Schwahn U, Jelenik T, Roden M, et al. BMP4 and BMP7 induce the white-to-brown transition of primary human adipose stem cells. Am J Physiol Physiol. (2014) 306:C43140. doi: 10.1152/ajpcell.00290.2013

32. Qian S-W, Tang Y, Li X, Liu Y, Zhang Y-Y, Huang H-Y, et al. BMP4mediated brown fat-like changes in white adipose tissue alter glucose and energy homeostasis. Proc Natl Acad Sci USA. (2013) 110:E798807. doi: 10.1073/pnas.1215236110

33. Tseng Y, Kokkotou E, Schulz T, Huang T, Winnay J, Taniguchi C, et al. New role of bone morphogenetic protein 7 in brown adipogenesis and energy expenditure. Nature. (2008) 454:1000-4. doi: 10.1038/nature07221

34. Whittle AJ, Carobbio S, Martins L, Slawik M, Hondares E, Vázquez MJ, et al. BMP8B increases brown adipose tissue thermogenesis through both central and peripheral actions. Cell. (2012) 149:871-85. doi: 10.1016/j.cell.2012.02.066

35. Pellegrinelli V, Peirce VJ, Howard L, Virtue S, Türei D, Senzacqua M, et al. Adipocyte-secreted BMP8b mediates adrenergic-induced remodeling of the neuro-vascular network in adipose tissue. Nat Commun. (2018) 9:1-18. doi: 10.1038/s41467-018-07453-x

36. Fisher FM, Kleiner S, Douris N, Fox EC, Mepani RJ, Verdeguer F, et al. FGF21 regulates PGC- $1 \alpha$ and browning of white adipose tissues in adaptive thermogenesis. Genes Dev. (2012) 26:271-81. doi: 10.1101/gad.177857.111

37. Chen MZ, Chang JC, Zavala-Solorio J, Kates L, Thai M, Ogasawara A, et al. FGF21 mimetic antibody stimulates UCP1-independent brown fat thermogenesis via FGFR1/קKlotho complex in non-adipocytes. Mol Metab. (2017) 6:1454-67. doi: 10.1016/j.molmet.2017.09.003

38. Zhang $\mathrm{Y}$, Xie C, Wang $\mathrm{H}$, Foss RM, Clare M, George EV, et al. Irisin exerts dual effects on browning and adipogenesis of human white adipocytes. Am J Physiol Endocrinol Metab. (2016) 311:E53041. doi: 10.1152/ajpendo.00094.2016

39. Elsen M, Raschke S, Eckel J. Browning of white fat: does irisin play a role in humans? J Endocrinol. (2014) 222:R25-38. doi: 10.1530/JOE-14-0189

40. Arhire LI, Mihalache L, Covasa M. Irisin: a hope in understanding and managing obesity and metabolic syndrome. Front Endocrinol. (2019) 10:524. doi: 10.3389/fendo.2019.00524

41. Seiler SE, Xu D, Ho J-P, Lo KA, Buehrer BM, Ludlow YJW, et al. Characterization of a primary brown adipocyte culture system derived from human fetal interscapular fat. Adipocyte. (2015) 4:303-10. doi: 10.1080/21623945.2015.1042192

42. Zilberfarb V, Piétri-Rouxel F, Jockers R, Krief S, Delouis C, Issad T, et al. Human immortalized brown adipocytes express functional beta3adrenoceptor coupled to lipolysis. J Cell Sci. (1997) 110:801-7.

43. Shinoda K, Luijten IHN, Hasegawa Y, Hong H, Sonne SB, Kim M, et al. Genetic and functional characterization of clonally derived adult human brown adipocytes. Nat Med. (2015) 21:389-94. doi: 10.1038/nm.3819

44. Xue R, Lynes MD, Dreyfuss JM, Shamsi F, Schulz TJ, Zhang H, et al. Clonal analyses and gene profiling identify genetic biomarkers of the thermogenic potential of human brown and white preadipocytes. Nat Med. (2015) 21:7608. doi: 10.1038/nm.3881

45. Markussen LK, Isidor MS, Breining P, Andersen ES, Rasmussen NE, Petersen LI, et al. Characterization of immortalized human brown and white pre-adipocyte cell models from a single donor. PLoS ONE. (2017) 12:e0185624. doi: 10.1371/journal.pone.0185624

46. Kishida T, Ejima A, Yamamoto K, Tanaka S, Yamamoto T, Mazda O. Reprogrammed functional brown adipocytes ameliorate insulin resistance and dyslipidemia in diet-induced obesity and type 2 diabetes. Stem Cell Rep. (2015) 5:569. doi: 10.1016/j.stemcr.2015.08.007

47. Takeda Y, Harada Y, Yoshikawa T, Dai P. Direct conversion of human fibroblasts to brown adipocytes by small chemical compounds. Sci Rep. (2017) 7:4304. doi: 10.1038/s41598-017-04665-x

48. Nedergaard J, Petrovic N, Lindgren EM, Jacobsson A, Cannon B. PPAR $\gamma$ in the control of brown adipocyte differentiation. Biochim Biophys Acta Mol Basis Dis. (2005) 1740:293-304. doi: 10.1016/j.bbadis.2005.02.003

49. Crisan M, Casteilla L, Lehr L, Carmona M, Paoloni-Giacobino A, Yap S, et al. A reservoir of brown adipocyte progenitors in human skeletal muscle. Stem Cells. (2008) 26:2425-33. doi: 10.1634/stemcells.2008-0325

50. Ullah I, Subbarao RB, Rho GJ. Human mesenchymal stem cells - current trends and future prospective. Biosci Rep. (2015) 35:e0191. doi: 10.1042/BSR20150025

51. Elabd C, Chiellini C, Carmona M, Galitzky J, Cochet O, Petersen R, et al. Human multipotent adipose-derived stem cells differentiate into functional brown adipocytes. Stem Cells. (2009) 27:2753-60. doi: 10.1002/stem.200

52. Rashnonejad A, Ercan G, Gunduz C, Akdemir A, Tiftikcioglu YO. Comparative analysis of human UCB and adipose tissue derived mesenchymal stem cells for their differentiation potential into brown and white adipocytes. Mol Biol Rep. (2018) 45:233-44. doi: 10.1007/s11033-018-4156-1

53. Huang P-I, Chen Y-C, Chen L-H, Juan C-C, Ku H-H, Wang S-T, et al. PGC-1 $\alpha$ mediates differentiation of mesenchymal stem cells to brown adipose cells. $J$ Atheroscler Thromb. (2011) 18:966-80. doi: 10.5551/jat.7401

54. Clevers H. Modeling development and disease with organoids. Cell. (2016) 165:1586-97. doi: 10.1016/j.cell.2016.05.082

55. Takahashi K, Tanabe K, Ohnuki M, Narita M, Ichisaka T, Tomoda K, et al. Induction of pluripotent stem cells from adult human fibroblasts by defined factors. Cell. (2007) 131:861-72. doi: 10.1016/j.cell.2007.11.019

56. Ahfeldt T, Schinzel RT, Lee Y-K, Hendrickson D, Kaplan A, Lum $\mathrm{DH}$, et al. Programming human pluripotent stem cells into white and brown adipocytes. Nat Cell Biol. (2012) 14:209-19. doi: 10.1038/ncb 2411

57. Lamarche É, Lala-Tabbert N, Gunanayagam A, St-Louis C, Wiper-Bergeron N. Retinoic acid promotes myogenesis in myoblasts by antagonizing transforming growth factor-beta signaling via C/EBP $\beta$. Skelet Muscle. (2015) 5:8. doi: 10.1186/s13395-015-0032-z 
58. Pawlowski M, Ortmann D, Bertero A, Tavares JM, Pedersen RA, Vallier L, et al. Inducible and deterministic forward programming of human pluripotent stem cells into neurons, skeletal myocytes, and oligodendrocytes. Stem Cell Rep. (2017) 8:803. doi: 10.1016/j.stemcr.2017.02.016

59. Moreau T, Evans AL, Vasquez L, Tijssen MR, Yan Y, Trotter MW, et al. Large-scale production of megakaryocytes from human pluripotent stem cells by chemically defined forward programming. Nat Commun. (2016) 7:11208. doi: $10.1038 /$ ncomms 11208

60. Zhang Y, Pak C, Han Y, Ahlenius H, Zhang Z, Chanda S, et al. Rapid singlestep induction of functional neurons from human pluripotent stem cells. Neuron. (2013) 78:785-98. doi: 10.1016/j.neuron.2013.05.029

61. Mohsen-Kanson T, Hafner A-L, Wdziekonski B, Takashima Y, Villageois P, Carrière A, et al. Differentiation of human induced pluripotent stem cells into brown and white adipocytes: role of pax3. Stem Cells. (2014) 32:145967. doi: 10.1002/stem.1607

62. Hafner A-L, Contet J, Ravaud C, Yao X, Villageois P, Suknuntha K, et al. Brown-like adipose progenitors derived from human induced pluripotent stem cells: identification of critical pathways governing their adipogenic capacity. Sci Rep. (2016) 6:32490. doi: 10.1038/srep32490

63. van Meeteren LA, ten Dijke P. Regulation of endothelial cell plasticity by TGF- $\beta$. Cell Tissue Res. (2012) 347:177-86. doi: 10.1007/s00441-011-1222-6

64. Nishio M, Yoneshiro T, Nakahara M, Suzuki S, Saeki K, Hasegawa M, et al. Production of functional classical brown adipocytes from human pluripotent stem cells using specific hemopoietin cocktail without gene transfer. Cell Metab. (2012) 16:394-406. doi: 10.1016/j.cmet.2012.08.001

65. Oka M, Kobayashi N, Matsumura K, Nishio M, Saeki K. Exogenous cytokinefree differentiation of human pluripotent stem cells into classical brown adipocytes. Cells. (2019) 8:373. doi: 10.3390/cells8040373

66. Pope BD, Warren CR, Parker KK, Cowan CA. Microenvironmental control of adipocyte fate and function. Trends Cell Biol. (2016) 26:74555. doi: 10.1016/j.tcb.2016.05.005

67. Sun K, Tordjman J, Clément K, Scherer PE. Fibrosis and adipose tissue dysfunction. Cell Metab. (2013) 18:470-7. doi: 10.1016/j.cmet.2013.06.016

68. Shoham N, Gefen A. Mechanotransduction in adipocytes. J Biomech. (2012) 45:1-8. doi: 10.1016/j.jbiomech.2011.10.023

69. Chun T-H, Hotary KB, Sabeh F, Saltiel AR, Allen ED, Weiss SJ. A pericellular collagenase directs the 3-dimensional development of white adipose tissue. Cell. (2006) 125:577-91. doi: 10.1016/j.cell.2006.02.050

70. Unser AM, Tian Y, Xie Y. Opportunities and challenges in threedimensional brown adipogenesis of stem cells. Biotechnol Adv. (2015) 33:96279. doi: 10.1016/j.biotechadv.2015.07.005

71. Murphy CS, Liaw L, Reagan MR. In vitro tissue-engineered adipose constructs for modeling disease. BMC Biomed Eng. (2019) 1:27. doi: 10.1186/s42490-019-0027-7

72. Pellegrinelli V, Heuvingh J, du Roure O, Rouault C, Devulder A, Klein C, et al. Human adipocyte function is impacted by mechanical cues. J Pathol. (2014) 233:183-95. doi: 10.1002/path.4347

73. Harms MJ, Li Q, Lee S, Zhang C, Kull B, Hallen S, et al. Mature human white adipocytes cultured under membranes maintain identity, function, and can transdifferentiate into brown-like adipocytes. Cell Rep. (2019) 27:21325.e5. doi: 10.1016/j.celrep.2019.03.026

74. Muller S, Ader I, Justine C, Leménager H, Achard P, Casteilla L, et al. Human adipose stromal-vascular fraction self-organizes to form vascularized adipose tissue in 3D cultures. (2019) 9:7520. doi: 10.1038/s41598-019-43624-6

75. Daquinag AC, Souza GR, Kolonin MG. Adipose tissue engineering in threedimensional levitation tissue culture system based on magnetic nanoparticles. Tissue Eng Part C Methods. (2013) 19:336-44. doi: 10.1089/ten.tec.2012.0198

76. Klingelhutz AJ, Gourronc FA, Chaly A, Wadkins DA, Burand AJ, Markan KR, et al. Scaffold-free generation of uniform adipose spheroids for metabolism research and drug discovery. Sci Rep. (2018) 8:523. doi: 10.1038/s41598-017-19024-Z

77. Unser AM, Mooney B, Corr DT, Tseng YH, Xie Y. 3D brown adipogenesis to create "Brown-Fat-in-Microstrands." Biomaterials. (2016). 75:123-34. doi: 10.1016/j.biomaterials.2015.10.017

78. Kuss M, Kim J, Qi D, Wu S, Lei Y, Chung S, et al. Effects of tunable, 3Dbioprinted hydrogels on human brown adipocyte behavior and metabolic function. Acta Biomater. (2018) 71:486-95. doi: 10.1016/j.actbio.2018.03.021

79. Louis F, Pannetier P, Souguir Z, Le Cerf D, Valet P, Vannier J-P, et al. A biomimetic hydrogel functionalized with adipose ECM components as a microenvironment for the 3D culture of human and murine adipocytes. Biotechnol Bioeng. (2017) 114:1813-24. doi: 10.1002/bit.26306

80. Bagchi M, Kim LA, Boucher J, Walshe TE, Kahn CR, D’Amore PA. Vascular endothelial growth factor is important for brown adipose tissue development and maintenance. FASEB J. (2013) 27:3257-71. doi: 10.1096/fj.12-221812

81. Sun K, Kusminski CM, Luby-Phelps K, Spurgin SB, An YA, Wang QA, et al. Brown adipose tissue derived VEGF-A modulates cold tolerance and energy expenditure. Mol Metab. (2014) 3:474-83. doi: 10.1016/j.molmet.2014.03.010

82. Shimizu I, Aprahamian T, Kikuchi R, Shimizu A, Papanicolaou KN, MacLauchlan S, et al. Vascular rarefaction mediates whitening of brown fat in obesity. J Clin Invest. (2014) 124:2099-112. doi: 10.1172/JCI7 1643

83. Min SY, Kady J, Nam M, Rojas-Rodriguez R, Berkenwald A, $\mathrm{Kim} \mathrm{JH}$, et al. Human "brite/beige" adipocytes develop from capillary networks, and their implantation improves metabolic homeostasis in mice. Nat Med. (2016) 22:312-8. doi: 10.1038/nm. 4031

Conflict of Interest: The authors declare that the research was conducted in the absence of any commercial or financial relationships that could be construed as a potential conflict of interest.

Copyright (c) 2020 Samuelson and Vidal-Puig. This is an open-access article distributed under the terms of the Creative Commons Attribution License (CC BY). The use, distribution or reproduction in other forums is permitted, provided the original author(s) and the copyright owner(s) are credited and that the original publication in this journal is cited, in accordance with accepted academic practice. No use, distribution or reproduction is permitted which does not comply with these terms. 